\title{
On the Study of Oscillating Viscous Flows by Using the Adomian-Padé Approximation
}

\author{
Chi-Min Liu ${ }^{1,2}$ \\ ${ }^{1}$ Division of Mathematics, General Education Center, Chienkuo Technology University, Changhua City 500, Taiwan \\ ${ }^{2}$ International Wave Dynamics Research Center, National Cheng Kung University, Tainan 701, Taiwan \\ Correspondence should be addressed to Chi-Min Liu; cmliu@ctu.edu.tw \\ Received 8 November 2014; Accepted 2 April 2015 \\ Academic Editor: Charalampos Tsitouras \\ Copyright (C) 2015 Chi-Min Liu. This is an open access article distributed under the Creative Commons Attribution License, which \\ permits unrestricted use, distribution, and reproduction in any medium, provided the original work is properly cited.

\begin{abstract}
The Adomian-Padé technique is applied to examine two oscillating viscous flows, the Stokes' second problem and the pressuredriven pulsating flow. Main purposes for studying oscillating flows are not only to verify the accuracy of the approximation solution, but also to provide a basis for analyzing more problems by the present method with the help of Fourier analysis. Results show that the Adomian-Padé approximation presents a very excellent behavior in comparison with the exact solution of Stokes' second problem. For the pulsating flow, only the Adomian decomposition method is required to perform the calculation as the fluid domain is finite where the Padé approximant may not provide a better solution. Based on present results, more problems can be mathematically solved by using the Adomian-Padé technique, the Fourier analysis, and powerful computers.
\end{abstract}

\section{Introduction}

The Adomian decomposition method has been widely studied and applied to solve mathematical problems [1]. It provides an efficient way to study a rather wide class of nonlinear as well as stochastic equations without linearization, perturbation, closure approximation, or discretization methods [2]. The main idea of the method is to decompose the key variable, say, $u$, into an infinite series of $u_{n}(n=0,1,2, \ldots)$ without requirements of weak nonlinearity and small perturbation. The first decomposed term $u_{0}$ is determined by the given initial or boundary conditions. Then other decomposed terms can be calculated by applying the integral operators to the governing equation with the help of lower-order terms. In recent years, there has been a great amount of efforts on applying the Adomian decomposition method have been applied to many fields [3-8] and some improvements of algorithms were also presented [9-12].

As all decomposed terms except the first one are calculated by integration, the solution is usually expressed in a form of a polynomial of the variable of integration (the temporal or spatial variable). Therefore, the applicable range of the Adomian decomposed solution will diverge quickly while this integration variable grows. To overcome the weakness, the Padé approximation is adopted to improve the accuracy of the solution. The main idea of Padé approximant is to transfer the original polynomial into a rational function of the order $[m / n]$ approximant [13]. In comparison of the form of Taylor series, Padé approximant usually gives a larger valid range of the derived solution. The Padé approximation has been widely adopted to improve the accuracy of the Adomian decomposed solution [14-16].

In this paper two cases of oscillating viscous flows will be investigated by using the Adomian-Padé approximation. The main reason why we study oscillating flows is that the present study can provide a basis for analyzing more problems while applying the Fourier analysis. The organization of this paper is as follows. In Section 2, Stokes' second problem describing the flow induced by an oscillating plate below is examined. Boundary conditions required for calculating the velocity evolution of the whole domain are the velocity and its gradient at the plate. The derived solution is compared with the exact solution for verifying its validity. In Section 3 the pressure-driven pulsating flow between two infinite parallel plates is calculated by giving the oscillating pressure gradient along the flow direction. The velocity at the center plane is given as the beginning condition. Results in Sections 2 and 3 
show very excellent behaviors in comparison with their exact solutions. Conclusion remarks are made in Section 4.

\section{Stokes' Second Problem}

A viscous flow generated by an oscillating plate below is the well-known Stokes' second problem. The plate is located at the plane $y=0$ and oscillates with the velocity $u_{0} \cos t \omega$ in its own plane. The kinematic viscosity of the fluid in the domain $y \geq 0$ is $\nu$. The corresponding dimensionless variables are assigned by

$$
\begin{aligned}
& U=\frac{u}{u_{0}}, \\
& Y=y \sqrt{\frac{\omega}{v}}, \\
& T=t \omega,
\end{aligned}
$$

where $U$ is the velocity, $Y$ the spatial parameter, and $T$ the time. Hence the problem can be described with dimensionless governing equation

$$
\frac{\partial U}{\partial T}=\frac{\partial^{2} U}{\partial Y^{2}}
$$

and boundary conditions

$$
\begin{gathered}
U(Y=0, T)=\cos T, \\
U(Y=\infty, T)=0 .
\end{gathered}
$$

The exact solution for (1) to (3) is (see $[17,18]$ for details)

$$
U=\exp \left(-\frac{Y}{\sqrt{2}}\right) \cos \left(T-\frac{Y}{\sqrt{2}}\right) .
$$

It is noted that if the initial condition, $U(Y, T=0)=0$, is considered in this problem, the corresponding solution will possess both steady-state and transient parts shown as [17]

$$
\begin{aligned}
U & =\frac{1}{4} \cdot e^{i T}\left\{\exp (-\sqrt{i} Y) \operatorname{erfc}\left(\frac{Y}{2 \sqrt{T}}-\sqrt{i T}\right)\right. \\
& \left.+\exp (\sqrt{i} Y) \operatorname{erfc}\left(\frac{Y}{2 \sqrt{T}}+\sqrt{i T}\right)\right\}+\frac{1}{4} \\
& \cdot e^{-i T}\left\{\exp (-\sqrt{-i} Y) \operatorname{erfc}\left(\frac{Y}{2 \sqrt{T}}-\sqrt{-i T}\right)\right. \\
& \left.+\exp (\sqrt{-i} Y) \operatorname{erfc}\left(\frac{Y}{2 \sqrt{T}}+\sqrt{-i T}\right)\right\},
\end{aligned}
$$

where $\operatorname{erfc}(z)$ denotes the complementary error function with the complex argument.

Now the Adomian-Padé method is applied to solve the problem. First we define the operator

$$
L_{Y}(\cdot) \equiv \frac{\partial^{2}}{\partial Y^{2}}(\cdot) \text {. }
$$

Hence (2) can be represented as

$$
L_{Y} U=\frac{\partial U}{\partial T}
$$

Applying the inverse operator

$$
L_{Y}^{-1}(\cdot)=\int_{0}^{Y} \int_{0}^{Y}(\cdot) \mathrm{d} Y \mathrm{~d} Y
$$

to (7) yields

$$
U(Y, T)=U(0, T)+U_{Y}(0, T) \cdot Y+\int_{0}^{Y} \int_{0}^{Y} \frac{\partial U}{\partial T} d Y d Y
$$

The first two terms in the right-hand side of (9) contain two boundary conditions at the plate $(Y=0)$. They play an important role while applying the beginning conditions of the Adomian approximation. They are given by (4) and its derivative

$$
\begin{aligned}
U(0, T) & =\cos T, \\
U_{Y}(0, T) & =-\cos \left(T+\frac{\pi}{4}\right) .
\end{aligned}
$$

Note that another boundary condition $U(\infty, T)=0$ which is not used in the Adomian analysis will be regarded as a condition for examining the accuracy of the derived solution. Now the velocity $U$ is assumed to be

$$
U(Y, T)=\sum_{n=0}^{\infty} U_{n}(Y, T) .
$$

Substituting (11) into (9) gives the following relations:

$$
\begin{aligned}
U_{0} & =U(0, T)+U_{Y}(0, T) \cdot Y \\
& =\cos T-Y \cos \left(T+\frac{\pi}{4}\right), \\
U_{n+1} & =\int_{0}^{Y} \int_{0}^{Y} \frac{\partial U_{n}}{\partial T} \mathrm{~d} Y \mathrm{~d} Y, \quad n \geq 1 .
\end{aligned}
$$

By calculating (13) term by term, we have

$$
\begin{aligned}
& U_{1}=-\frac{Y^{2}}{2 !} \sin T+\frac{Y^{3}}{3 !} \sin \left(T+\frac{\pi}{4}\right), \\
& U_{2}=-\frac{Y^{4}}{4 !} \cos T+\frac{Y^{5}}{5 !} \cos \left(T+\frac{\pi}{4}\right), \\
& U_{3}=+\frac{Y^{6}}{6 !} \sin T-\frac{Y^{7}}{7 !} \sin \left(T+\frac{\pi}{4}\right), \\
& U_{4}=+\frac{Y^{8}}{8 !} \cos T-\frac{Y^{9}}{9 !} \cos \left(T+\frac{\pi}{4}\right),
\end{aligned}
$$

The coefficients of Padé [2/2] solution can be determined by balancing $U_{04}=\sum_{n=0}^{4} U_{n}$ and

$$
U[2 / 2]=\frac{p_{0}+p_{1} Y+p_{2} Y^{2}}{1+q_{1} Y+q_{2} Y^{2}} .
$$




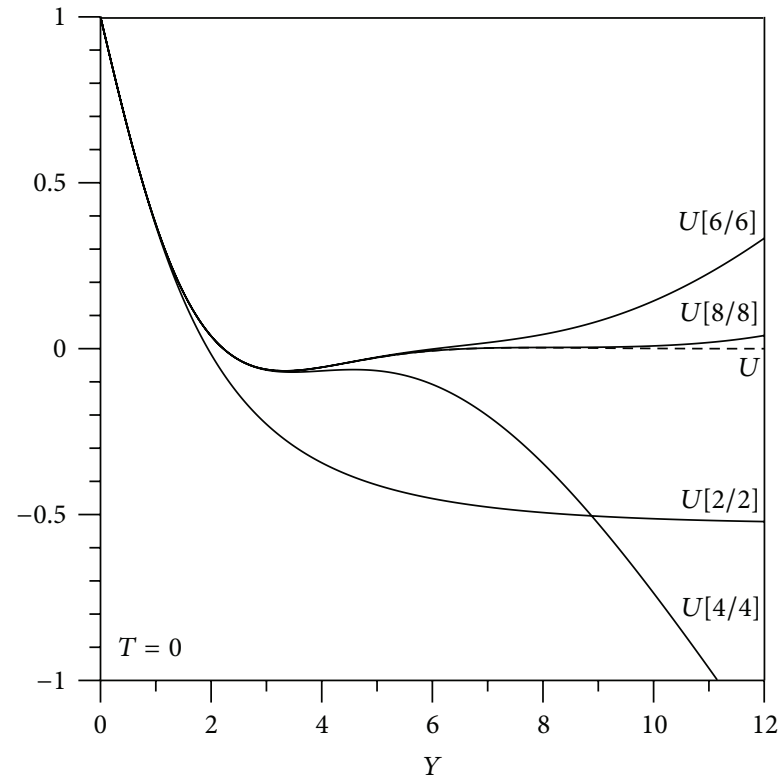

Figure 1: Comparison of Padé $[n / n]$ solutions (solid lines) and exact solution (dash line) for Stokes' second problem at $T=0$.

The results are

$$
\begin{aligned}
& p_{0}=\cos T \\
& p_{1}=\frac{5 \cos T-\cos 3 T-15 \sin T+\sin 3 T}{4 \sqrt{2}(-3+\cos 2 T)} \\
& p_{2}=-\frac{\sin T}{12}+\frac{\cos T+4 \sin T}{6(-3+\cos 2 T)} \\
& q_{1}=\frac{-3 \sqrt{2}+2 \cos (2 T+\pi / 4)}{4(-3+\cos 2 T)} \\
& q_{2}=\frac{4+\sin 2 T}{12(3-\cos 2 T)}
\end{aligned}
$$

By following similar processes shown above, higher-order Padé approximants can be readily obtained with the help of commercial software (e.g., Mathematica) and the details are neglected herein. Figure 1 shows velocity profiles calculated by the Adomian-Padé [2/2], [4/4], [6/6], and [8/8] solutions and by the exact solution (4) for the case $T=0$. Solid and dash lines indicate the Padé $[n / n]$ solutions and the exact solution, respectively. It is seen that the higher-order Adomian-Padé solution will result in a more accurate behavior in comparison with the exact solution. Due to rapid and great progress of calculating ability of computers, the applicable domain in $Y$ will be efficiently expanded by much higher-order Padé solutions. Solutions for $T=\pi / 2$ are plotted in Figure 2. Results are similar to those demonstrated for Figure 1.

The present method can play an important role in practical cases. For example, if the boundary conditions at the plate can be measured by current meters or other facilities, velocity profile at any elevation $Y$ can be mathematically calculated without using the equipment to measure the flow speed at the desired place.

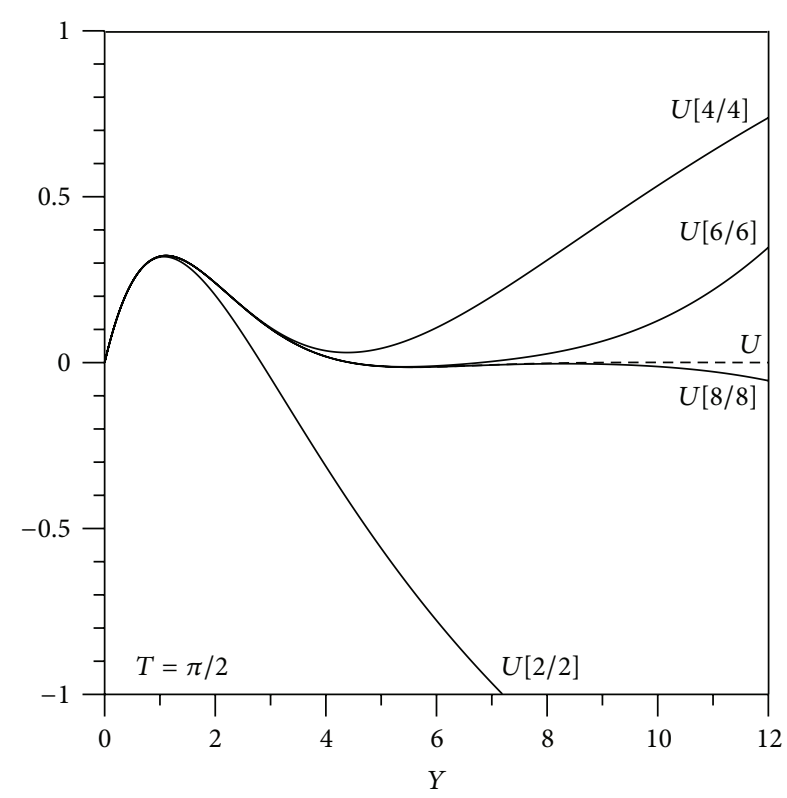

Figure 2: Comparison of Padé $[n / n]$ solutions (solid lines) and exact solution (dash line) for Stokes' second problem at $T=\pi / 2$.

\section{Pressure-Driven Pulsating Flow}

Another oscillating flow, the pressure-driven pulsating flow, is studied in this section. This flow describes that a fluid bounded by two parallel plates located at $y= \pm a$ is driven by a time-dependent pressure gradient. The governing equation is

$$
\frac{\partial u}{\partial t}=-\frac{1}{\rho} \frac{\partial p}{\partial x}+\nu \frac{\partial^{2} u}{\partial y^{2}}
$$

where $p$ is the pressure and

$$
\frac{\partial p}{\partial x}=p_{0} \cos t \omega
$$

is given. By introducing the following dimensionless variables:

$$
\begin{aligned}
U & =\frac{u}{a \omega}, \\
Y & =\frac{y}{a}, \\
T & =t \omega,
\end{aligned}
$$

(17) can be rewritten as

$$
\frac{\partial U}{\partial T}=-\left(\frac{p_{0}}{\rho \omega^{2} a}\right) \cos T+\left(\frac{v}{a^{2} \omega}\right) \frac{\partial^{2} U}{\partial Y^{2}},
$$

where $p_{0} / \rho \omega^{2} a=1$ and $\nu / a^{2} \omega=1$ are assigned hereafter for the purpose of simplification. The boundary conditions at both plates are

$$
U(Y= \pm 1, T)=0 .
$$


The exact solution for (20) and (21) is (see [18] for details)

$$
\begin{array}{r}
U=\operatorname{Re}\left\{i e^{i T}\left[1-\frac{\cosh ((1+i) Y / \sqrt{2})}{\cosh ((1+i) 1 / \sqrt{2})}\right]\right\}, \\
-1 \leq Y \leq 1,
\end{array}
$$

where Re denotes the real part of the complex. The velocity and its gradient at the plane $Y=0$ will be adopted as the beginning conditions. They are

$$
\begin{aligned}
U(0, T)= & \sin T\left[\frac{2 \cosh \sqrt{1 / 2} \cos \sqrt{1 / 2}}{\cosh \sqrt{2}+\cos \sqrt{2}}-1\right] \\
& -\cos T\left[\frac{2 \sinh \sqrt{1 / 2} \sin \sqrt{1 / 2}}{\cosh \sqrt{2}+\cos \sqrt{2}}\right], \\
U_{Y}(0, T)= & 0,
\end{aligned}
$$

where the former is obtained from (22) and the latter indicates that the flow is symmetric with respect to $Y=0$. It is also noted that boundary conditions at both plates will be seen as a condition for verifying the accuracy of the derived solution. Applying (8) to (20) with the help of (23) leads to

$$
U(Y, T)=U(0, T)+\frac{Y^{2}}{2} \cos T+\int_{0}^{Y} \int_{0}^{Y} \frac{\partial U}{\partial T} \mathrm{~d} Y \mathrm{~d} Y
$$

Now we substitute (11) into (24); the result is

$$
\begin{gathered}
U_{0}=U(0, T)+\frac{Y^{2}}{2 !} \cos T, \\
U_{1}=\frac{Y^{2}}{2 !} U_{T}(0, T)-\frac{Y^{4}}{4 !} \sin T, \\
U_{2}=\frac{Y^{4}}{4 !} U_{T T}(0, T)-\frac{Y^{6}}{6 !} \cos T, \\
U_{3}=\frac{Y^{6}}{6 !} U_{T T T}(0, T)+\frac{Y^{8}}{8 !} \sin T, \\
U_{4}=\frac{Y^{8}}{8 !} U_{T T T T}(0, T) T+\frac{Y^{10}}{10 !} \cos T,
\end{gathered}
$$

Using $U_{04}$ defined in previous section and balancing it with $U[2,2]$, the coefficients of $U[2,2]$ are solved:

$$
\begin{aligned}
& p_{0}=-\frac{\sin T(\cos \sqrt{2}-2 \cosh \sqrt{1 / 2} \cos \sqrt{1 / 2}+\cosh \sqrt{2})+2 \cos T \sin \sqrt{1 / 2} \sinh \sqrt{1 / 2}}{\cosh \sqrt{2}+\cos \sqrt{2}}, \\
& p_{1}=0, \\
& p_{2}=\frac{\sin T \sin \sqrt{1 / 2} \sinh \sqrt{1 / 2}+\cos T \cos \sqrt{1 / 2} \cosh \sqrt{1 / 2}}{\cosh \sqrt{2}+\cos \sqrt{2}} \\
& q_{1}=0, \\
& q_{2}=\frac{-\sin T \cos \sqrt{1 / 2} \cosh \sqrt{1 / 2}+\cos T \sin \sqrt{1 / 2} \sinh \sqrt{1 / 2}}{12(\cosh \sqrt{2}+\cos \sqrt{2})} .
\end{aligned}
$$

Figure 3 presents a comparison of different approximation solutions for the upper fluid domain $(0 \leq Y \leq 1)$. Ratios of $U[2 / 2]$ (solid lines) and $U_{04}$ (dash lines) to the exact solution $U$ are plotted for times $T=0$ (thin lines) and $T=\pi / 2$ (bold lines). It is seen that the polynomial solution $U_{04}$ behaves slightly better than the Padé [2/2] solution; namely, the former approximation is slightly closer to its exact solution when $Y$ approaches unity. The reason is that, in the range of $0 \leq Y \leq 1$, the Padé [2/2] solution may not give a more excellent simulation than the truncated polynomial solution $U_{04}$. This implies that higher-order Padé approximant may be not required to improve the accuracy of the polynomial solution for problems considered in a finite domain.

\section{Conclusions}

This paper presents the examination of Stokes' second problem and the pressure-driven pulsating flow by applying the Adomian-Padé technique. For Stokes' second problem, higher-order Adomian-Padé solution behaves very well in comparison with the exact solution while the spatial parameter grows. For pressure-driven pulsating flow, the Adomian approximation provides satisfactory results and the application of the Padé approximant may be unnecessary for the case of the finite domain. The above results demonstrate that the method used in this paper can be applied to solve more complicated problems with the help of the Fourier analysis and powerful computers. 


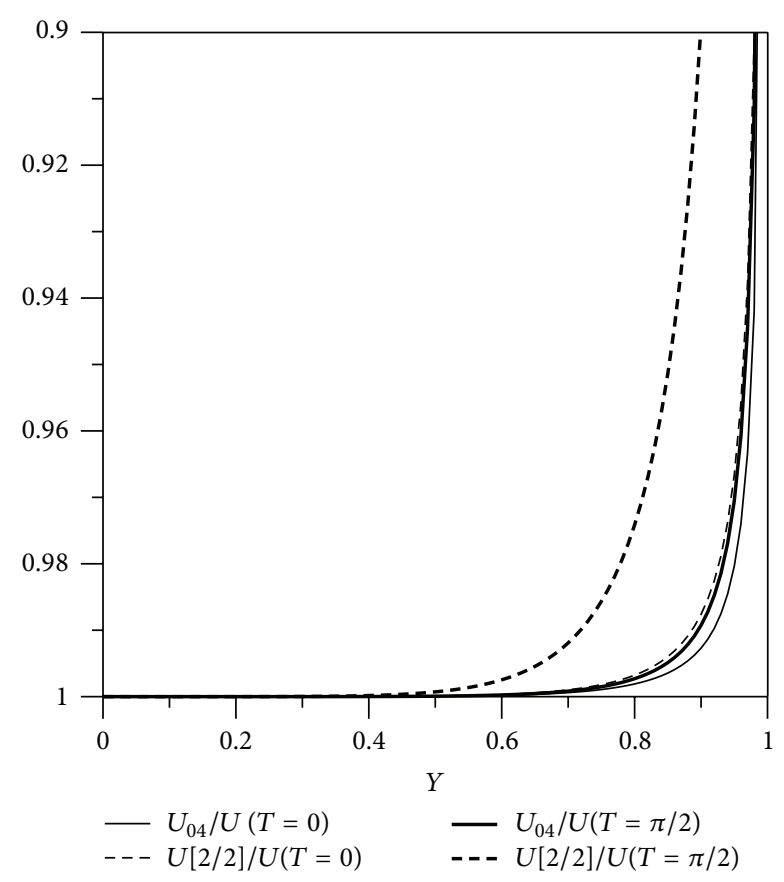

FIGURE 3: Ratios of polynomial solution and its Padé $[2,2]$ approximation to the exact solution for pressure-driven pulsating flow.

\section{Conflict of Interests}

The author declares that there is no conflict of interests regarding the publication of this paper.

\section{Acknowledgment}

Financial support from the Ministry of Science and Technology of Taiwan through Grants NSC 101-2221-E-270-001-MY2 and MOST 104-2911-I-006-301 is acknowledged.

\section{References}

[1] G. Adomian, Solving Frontier Problems of Physics: The Decomposition Method, vol. 60 of Fundamental Theories of Physics, Kluwer Academic, 1994.

[2] G. Adomian, "A review of the decomposition method in applied mathematics," Journal of Mathematical Analysis and Applications, vol. 135, no. 2, pp. 501-544, 1988.

[3] S. A. Khuri, "A Laplace decomposition algorithm applied to a class of nonlinear differential equations," Journal of Applied Mathematics, vol. 1, no. 4, pp. 141-155, 2001.

[4] S. M. El-Sayed and D. Kaya, "The decomposition method for solving $(2+1)$-dimensional Boussinesq equation and $(3+1)$ dimensional KP equation," Applied Mathematics and Computation, vol. 157, no. 2, pp. 523-534, 2004.

[5] D. J. Evans and K. R. Raslan, "The Adomian decomposition method for solving delay differential equation," International Journal of Computer Mathematics, vol. 82, no. 1, pp. 49-54, 2005.

[6] I. H. Abdel-Halim Hassan, "Comparison differential transformation technique with Adomian decomposition method for linear and nonlinear initial value problems," Chaos, Solitons and Fractals, vol. 36, no. 1, pp. 53-65, 2008.
[7] A. H. Bokhari, G. Mohammad, M. T. Mustafa, and F. D. Zaman, "Adomian decomposition method for a nonlinear heat equation with temperature dependent thermal properties," Mathematical Problems in Engineering, vol. 2009, Article ID 926086, 12 pages, 2009.

[8] A.-M. Wazwaz, R. Rach, and J.-S. Duan, "Adomian decomposition method for solving the Volterra integral form of the LaneEmden equations with initial values and boundary conditions," Applied Mathematics and Computation, vol. 219, no. 10, pp. 5004-5019, 2013.

[9] A.-M. Wazwaz, "A new algorithm for calculating Adomian polynomials for nonlinear operators," Applied Mathematics and Computation, vol. 111, no. 1, pp. 33-51, 2000.

[10] J.-S. Duan, "An efficient algorithm for the multivariable Adomian polynomials," Applied Mathematics and Computation, vol. 217, no. 6, pp. 2456-2467, 2010.

[11] J.-S. Duan, "Recurrence triangle for Adomian polynomials," Applied Mathematics and Computation, vol. 216, no. 4, pp. 12351241, 2010.

[12] P.-Y. Tsai and C.-K. Chen, "A new algorithm on the solutions of forced convective heat transfer in a semi-infinite flat plate," Journal of Mechanics, vol. 27, no. 1, pp. 63-69, 2011.

[13] G. A. Baker and P. Graves-Morris, Padé Approximants, Cambridge University Press, 2nd edition, 1996.

[14] A.-M. Wazwaz, "Analytical approximations and Pad approximants for Volterra's population model," Applied Mathematics and Computation, vol. 100, no. 1, pp. 13-25, 1999.

[15] T. A. Abassy, M. A. El-Tawil, and H. K. Saleh, "The solution of $\mathrm{KdV}$ and $\mathrm{mKdV}$ equations using Adomian Padé approximation," International Journal of Nonlinear Sciences and Numerical Simulation, vol. 5, no. 4, pp. 327-339, 2004.

[16] M. Dehghan, A. Hamidi, and M. Shakourifar, "The solution of coupled Burgers' equations using Adomian-Pade technique," Applied Mathematics and Computation, vol. 189, no. 2, pp. 10341047, 2007.

[17] C.-M. Liu and I.-C. Liu, "A note on the transient solution of Stokes' second problem with arbitrary initial phase," Journal of Mechanics, vol. 22, no. 4, pp. 349-354, 2006.

[18] I. G. Currie, Fundamental Mechanics of Fluids, McGraw-Hill, New York, NY, USA, 2nd edition, 1993. 


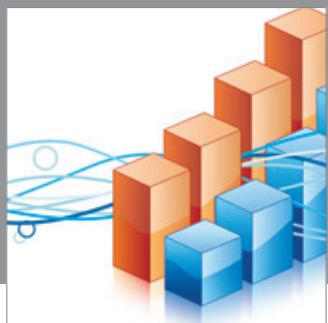

Advances in

Operations Research

mansans

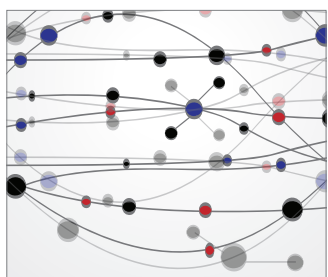

The Scientific World Journal
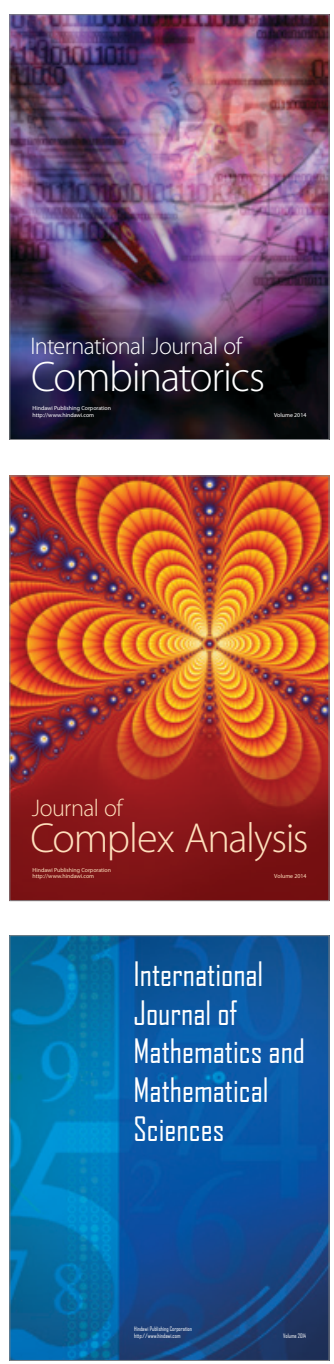
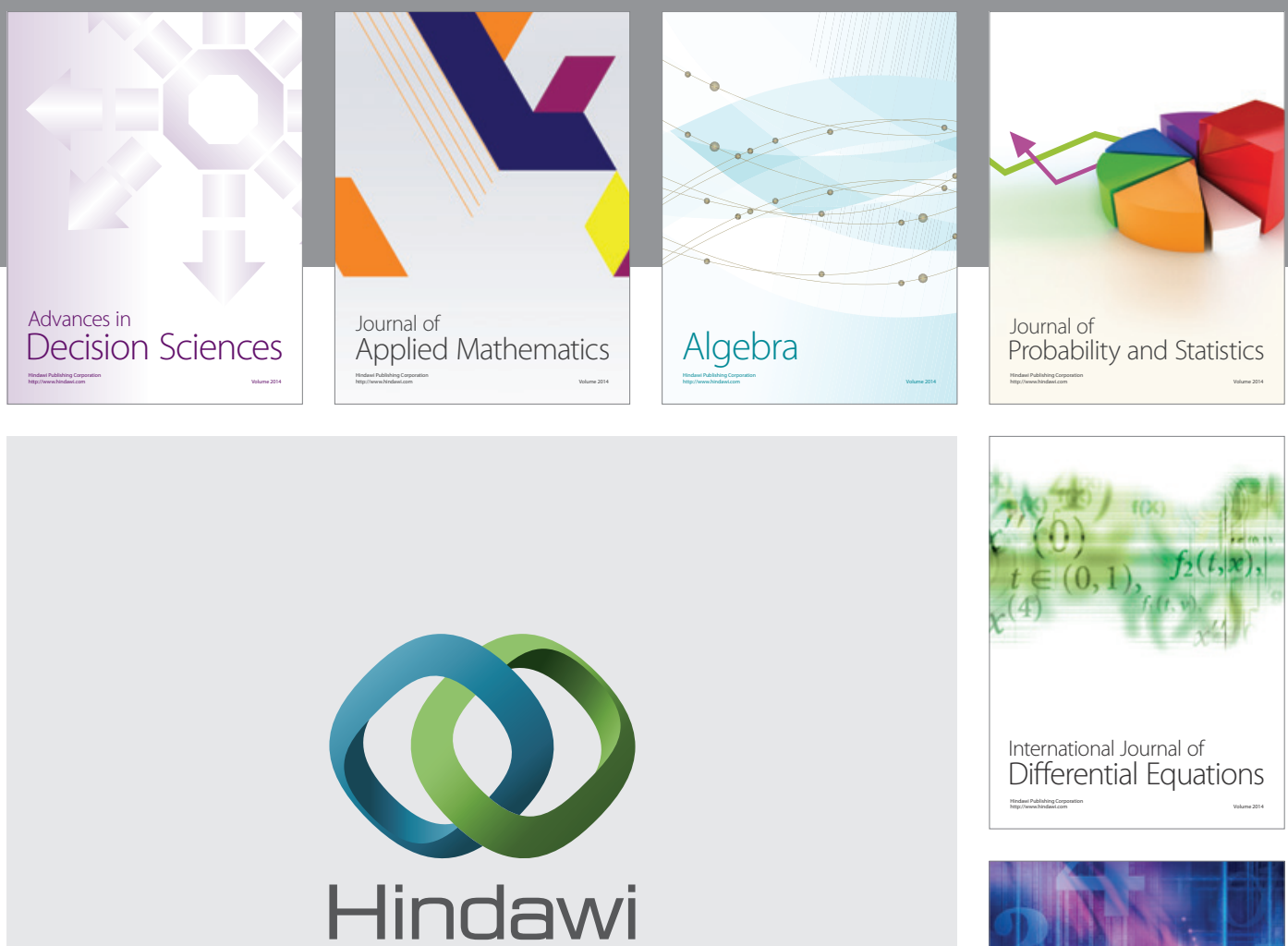

Submit your manuscripts at http://www.hindawi.com
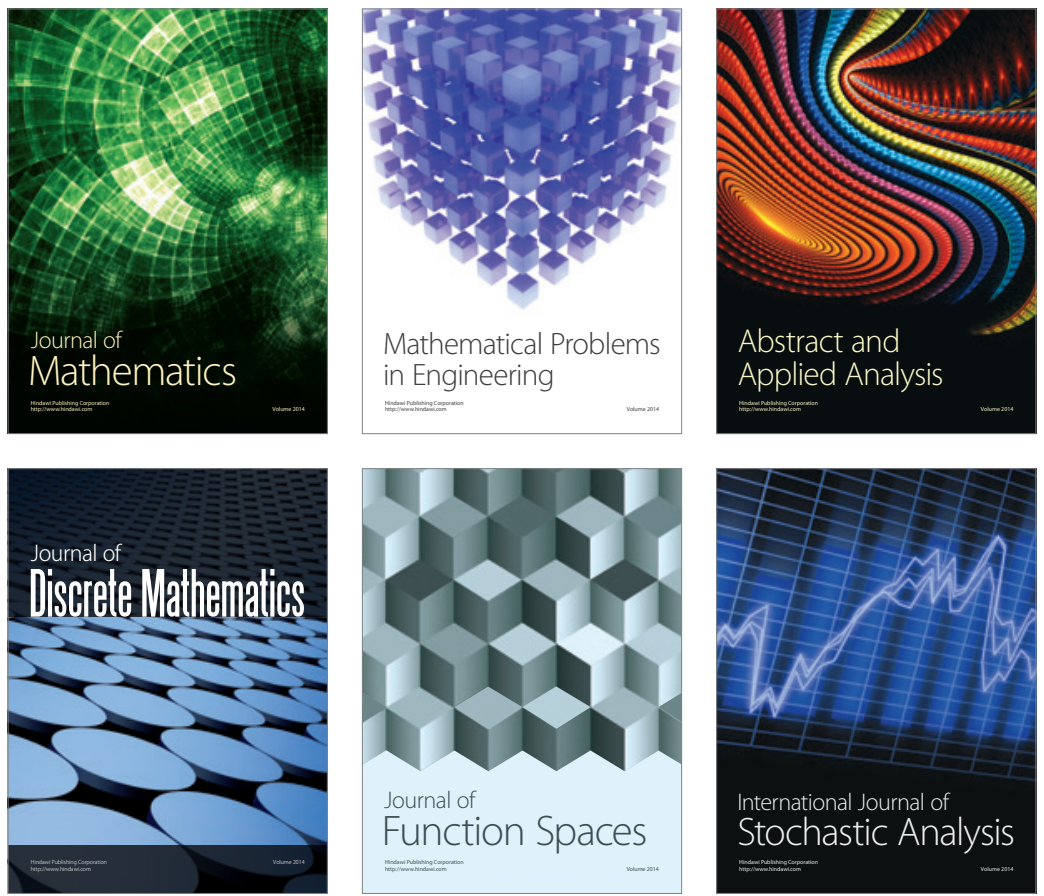

Journal of

Function Spaces

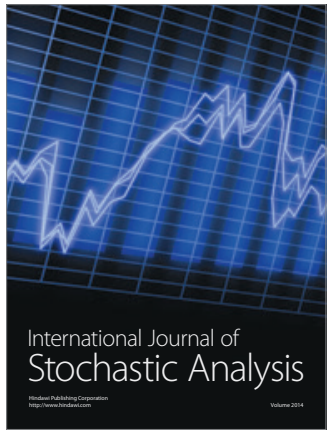

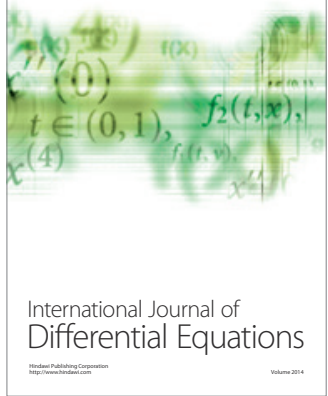
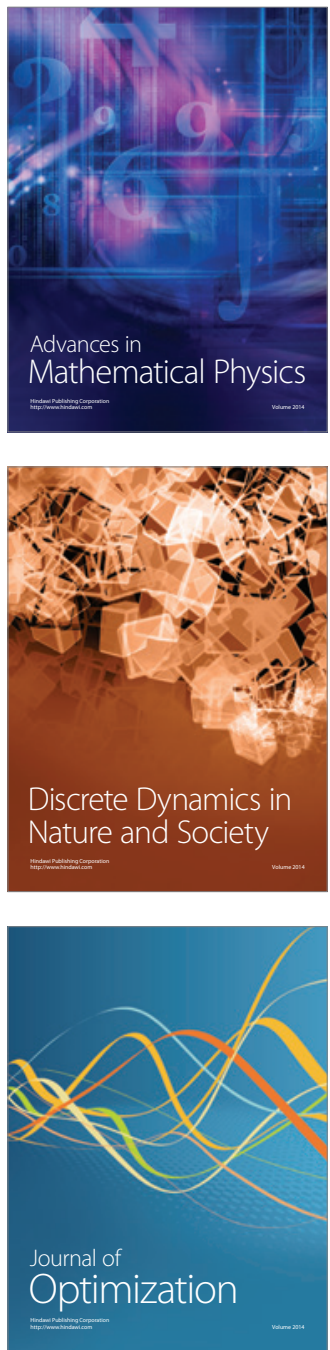